Capítulo 12

\title{
Perspectiva crítica y compromiso ético micropolítico: otros mundos posibles en la experiencia de investigación metodológica en comunicación
}

\section{DOI: https://doi.org/10.16921/ciespal.23.32}

Lisiane Machado Aguiar

Universidad Federal de Roraima-UFRR.

\section{Resumen}

En el campo académico de la comunicación en América Latina, la vertiente Mattelart presenta una orientación metodológica que parte de la crítica por medio de la experiencia ética y no de una dicotomía entre teoría y práctica. Así, el sujeto revolucionario en los escritos de Mattelart estaría muy próximo de la micropolítica y de la microfísica del poder, en relación a la crítica como una producción de subjetividad ética. En esa conexión, buscamos diálogos con la filosofía de la diferencia por medio de una genealogía del concepto de crítica acoplado con las metodologías de investigación. Luego para hacer una crítica hay que problematizar. Es posible pensar en ciertas formas de interrogación que conducen teórico-metodológicamente: ¿cómo el objeto de estudio, en un momento dado, puede ser problematizado a través de una relación entre el poder y ciertos estados mixtos del saber metodológico? Se trata, entonces, de modos de preguntar a cada formación histórica, creando un campo de posibilidades de donde emergen las problemáticas. En esta línea, la escritura del texto busca una estética 
como configuración de la experiencia, en que colectiviza lo aprehendido no a través de una sucesión cronológica, sino que intenta interrogar y narrar por otros modos. Los datos no se recopilan y almacenan, pero emergen a partir de lo que la investigación interroga.

Palabras-clave: crítica, ética, micropolítica, investigación metodológica.

\section{Introducción}

"Mattelart es un investigador incansable, su tenacidad científica y su compromiso ético revolucionario definen el comportamiento crítico y autocrítico" (Maldonado, 1999, p. 13, traducción propia). Empiezo con esa citación de Efendy Maldonado ${ }^{1}$, que fue mi maestro universitario de comunicación y cuyas enseñanzas sigo compartiendo, no solo en mis actividades académicas, sino en las experiencias de la vida. De igual forma, así como la vertiente Mattelart es para Maldonado una inspiración recurrente para la reflexión de la comunicación crítica y de una vida con compromiso ético revolucionario.

La experiencia llamada "vida" fue también un hilo conductor en las filosofías de la diferencia de Derrida, Foucault, Deleuze e Guattari que, con sus enseñanzas académicas y la militancia, marcaron el mítico mayo de 68, a través de sus críticas que intentaban pensar lo imposible como una forma de activismo ético, estético y político (Gutting, 2011). Fue en esa misma época de los años 60 que Mattelart salió de Bélgica con su doctorado de derecho rumbo a la Sorbonne para una especialización que le permitió, más tarde, ingresar como profesor en la Escuela de Sociología de la Universidad Católica de Chile, hasta el golpe de Estado fascista que, en 1973, le hizo retornar a Francia.

1 Catedrático/Investigador del Programa de Doctorado (Ph. D) en UNISINOS-Brasil. Autor de una expresiva producción científica bibliográfica sobre América Latina, epistemologías, teorías, metodologías en comunicación. Premios UNESCO, CAPES, INTERCOM, COMPÓS en investigación. Catedrático Titular- CIESPAL-Armand Mattelart. Coordinador General para América Latina de la Red AMLAT. 
De acuerdo con Maldonado y Fígaro (2020), la inmersión latinoamericana de Mattelart se diferencia de otros intelectuales que buscan "lucrar como reproductores del logocentrismo y del etnocentrismo eurocentrico, la dupla Mattelart llegó para aprender, trabajar, luchar, existir y amar los processos socioculturales, educativos, investigativos y constitutivos del pensamento comunicacional critico en el continente" (2020, p. 7, traducción propia).

Así como los Matterlarts se desterritorializaron al vivenciar las procesualidades decoloniales de una vida en Latinoamérica, otros intelectuales tuvieron la misma posibilidad de mezclar saberes. En Brasil, dentro del universo académico, es recurrente tener como referencia al portugués Boa Ventura de Sousa Santos, al pensar en "epistemologías del sur", la "descolonización del saber", las "ecologías de saberes" contra el "pensamiento abismal". Ese otro ejemplo, no es una crítica entre estar arriba o debajo de la línea del Ecuador, sino, más bien, una constatación de la importancia de las integraciones y colaboraciones para un desarrollo colectivo ${ }^{2}$. Boa Ventura de Sousa Santos (2000), en el libro Crítica de la Razón Indolente: contra el desperdicio de la experiencia, pregunta: ¿Por qué es un desafío producir una teoría crítica? Y pregunto desde una posición de profesora-latina-periférica: ¿Por qué es tan desafiador producir una investigación metodológica crítica en comunicación?

Por medio de esa problematización quiero conectar la investigación crítica de la comunicación en América Latina por medio de la vertiente Mattelart, en diálogo con la filosofía de la diferencia, en una genealogía del concepto de crítica en relación a las metodologías de investigación. Pero quiero hacerlo por medio de dos intercesores intelectuales que de forma antropofágica hicieron una interlocución epistémica metodológica: Efendy Maldonado con el concepto de crítica por medio de la vertiente de Michele+Armand Mattelart y Silvia

2 Solo para recordar, el proyecto llamado ALICE: Leading Europe to a New Way of Sharing the World Experiences es financiado por el Consejo Europeo de Investigación. El proyecto permitió reunir un equipo de jóvenes investigadores de diferentes países y líneas de investigación que surgieron de las premisas epistemológicas, teórico-analíticas y metodológicas de sus trabajos. Disponible en: https://alice.ces. uc.pt/en/index.php/homepage-2/ Acceso en: 02 de marzo de 2021. 
Rivera Cusicanqui con el concepto de micropolítica por medio del encuentro con Suely Rolnik+Deleuze e Guattari.

\section{Metodología de investigación: problematización ética, estética y política}

Metodología de investigación es una disciplina obligatoria en los cursos de postgrado del sistema stricto sensu en Brasil. Todavía, muchas obras sobre el asunto se limitan a discutir las etapas del proyecto sin buscar los sentidos constitutivos para pensar toda la investigación de forma procesual. Aquí propongo una estética de escritura rizomática y (po)ética: "caminante no hay camino, se hace camino al andar (...)" (Machado 1983).

A cada párrafo se busca la reanudación de cosas que no se acabaron en absoluto. Es necesario desvestirse de una lógica capitalista que busca la rapidez sucinta de respuestas, pues ellas van siendo construidas durante la lectura como una caminada.

Entonces, se hace del pensamiento un trabajo cooperativo, solidario y, quizá, educativo del sujeto-investigador y del que se depara con el escrito. Lejos de una pretensión de verdad totalizadora, es una ética de la caminada, que intenta expresar el pensar, por lo que exige de la persona que lee que también piense, siempre en busca de un sentido.

Cuando naturalizamos que el método en una investigación es sólo una meta para llegar a un camino, nos estamos aferrando a una etimología reduccionista de la palabra. Por otro lado, por medio de una perspectiva crítica sobre la genealogía de su epistemología permite una nueva vitalidad, un deseo colectivo de cuestionar nuestras formas de investigar y aprender a problematizar.

De acuerdo com Efendy (1999, p. 13, traducción propia), "Mattelart tiene una mirada metódica y es un cuestionador insistente de los elementos claves de los problemas, al mismo tiempo es un constructor de elementos cruciales de los problemas comunicativos". 
Mattelart presenta una orientación metodológica que parte de la crítica por medio de la experiencia ética y no de una dicotomía cuestionable entre teoría y práctica. El sujeto revolucionario de Mattelart estaría muy próximo de la micropolítica en relación a la crítica como una producción de subjetividad ética. La ética inspira un caminar crítico de los modelos teleológicos y permite pensar de manera diferente sobre las prácticas que influyen en los hechos.

Foucault (1995), en su ensayo cuestionador 'Qué es la crítica?', presenta una respuesta muy cerca de lo que materializa Mattelart (1981, 1991, 1994, 1996) al mirar para los desafíos de la comunicación: un pensamiento crítico que constituye el esfuerzo por reflexionar sobre el problema más allá del juicio moral universal. En este sentido, la crítica se hace desde el acto de cuestionar los límites de los modos de conocimiento ya establecidos y representa una posibilidad para una verdad que aún no se conoce o que aún no existe, la crítica

es el movimiento por el cual el sujeto se atribuye el derecho de interrogar la verdad por sus efectos de poder y al poder por sus discursos de verdad; pues bien, la crítica será el arte de la incertidumbre voluntaria, el de la indocilidad reflexiva. La crítica tendría esencialmente por función la desujeción en el juego de lo que se podría denominar, en una palabra, la política de la verdad (Foucault, 1995, p. 8).

Mattelar en el libro De Orwell al cibercontrol, escrito con André Vitalis toma como referencia la obra de Foucault Vigilar y castigar, pero el más interesante es como desarrolla la crítica de la hipervigilancia de los sujetos en las sociedades contemporáneas. Esa crítica busca las doctrinas y estrategias de seguridad en los procesos geopolíticos $(2015$, p. 5). Son esos procesos geopolíticos que conectan a una micropolítica como medio de ejercitar la crítica para pensar otros mundos posibles.

Silvia Rivera Cusicanqui, en su libro Un mundo ch'ixi es posible: ensayos desde un presente en crisis (2018), tiene un capítulo que se llama Micropolítica andina. Formas elementales de insurgencia cotidiana. Aquí narra su encuentro con Suely Rolnik y poco después leyó el trabajo 
de esa autora con Félix Guattari: Micropolítica. Cartografías del Deseo (2006). Para Guattari y Rolnik (1986, p. 133) "la cuestión de la micropolítica es de cómo reproducimos (o no), los modos de subjetivación dominante (...)" y para Silvia, "la micropolítica es un escapar permanente a los mecanismos de la política. Es constituir espacios por fuera del Estado, mantener en ellos un modo de vida alternativo, en acción, sin proyecciones teleológicas ni aspiraciones al cambio de estructuras" (2018, p. 142).

Es ese modo de vida alternativo que quiero proponer al hablar de la vertiente de los Mattelart, por médio de los estudios críticos que hace Efendy Maldonado en un bricolage de lo más ch'ixi, o sea mezclado.

Efendy Maldonado al escribir sobre la teoría crítica de los Mattelart desarrolla un pensamiento tanbién crítico sobre las metodológicas de la comunicación y propone una transmetodología que conjuga propuestas metodológicas mixtas que interrelacionen las construcciones conceptuales al enlace de lógicas diversas, tan pertinentes, para las problemáticas en comunicación (MALDONADO, 2008, 2006, 2003, 2002).

Pensar la 'problemática' en una investigación, que adopta la propuesta transmetodológica, es construir el 'problema de investigación' de forma micropolítica por medio de la contextualización del problema/objeto; de la investigación empírica y de la praxis teórica como medio de trabajar con los conceptos de forma crítica y renovadora. Así, es posible pensar que esas dimensiones al convergir juntas ayudan establecer una epistemología transmetodológica que es crítica para conjugar la praxis teórica y las estrategias metodológicas al carácter multicontextual de las investigaciones en comunicación.

"Construir teoría y metodología en comunicación, hoy, implica la realización de intensas inversiones de carácter cognitivo, lógico, vivencial, histórico y político." (Maldonado, 2002, s/ p., traducción propria). En el campo de la comunicación, nos damos cuenta de que ciertos modelos deterministas metodológicos están siendo ampliamente cuestionados, lo que permite diferentes formas de hacer investigación. Con esto, se constituyen una posibilidad de renovación crítica de lo teórico 
con las problematizaciones metodológicas, con las dimensiones empíricas del objeto y a la pluralidad del sujeto-investigador.

Con un pensamiento contrario a la lógica metafísica se producen diferentes modos de subjetivación: concibiendo la vida como relato, lo que significa que el sujeto humano es, como pensaba Proust (1998), un novelista de sí mismo. El proceso de autoconocimiento tiene que ver con diferentes momentos de la vida como un espacio de interlocución que educa al componer el relato del propio aprendizaje, de cómo se configuran y ayudan a prestar cuidadosa atención a la experiencia vivida y la de sus signos.

El buen escritor, dice Deleuze (1998), nunca escribe para convertirse en escritor, sino para subvertirse haciendo de la escritura más que escrita, serían “pasajes de vida”. También, sería algo que Rimbaud (1995) siempre profanaba: la de querer ser poeta y trabajar para volverse vidente. Para que el escritor/escritores(as) ejerzan la libertad se exige constantes diálogos con sus modos de existencia; es decir, "para que esa práctica de libertad tome forma en un éthos que sea bueno, bello, honroso, respetable, memorable y que pueda servir, es necesario todo un trabajo de sí sobre sí mismo" (Foucault, 2010, p. 270). En un trabajo de sí sobre sí mismo hay que preguntar: ¿cómo puedo constituirme a mí mismo como sujeto crítico?

Esta subversión instaura un problema político en las investigaciones que trabajan con crítica: ¿cómo regular relaciones entre individuos que piensan de modos divergentes? Si no hay valores morales racionales comprobados y universalmente compartidos ¿cómo asegurar que haya todavía una narrativa científica para el desarrollo metodológico en la academia? Sin macro-discursos se podría, entonces, ¿pensar en discursos menores? Sabiendo que el conocimiento científico sólo puede continuar en la medida en que se investiga, se puede preguntar: ¿cómo se busca metodológicamente en un proyecto de investigación hoy?

Así es necesario desterritorializar el pensar y causar un extrañamiento por médio de la crítica. Para eso es preciso desnaturalizar las suposiciones que hacen buscar respuestas enclavadas en las propias 
indagaciones. En esa medida es necesario ir más allá y considerar el presente contexto en el que se inserta el conjunto de problemas.

Pensando todos estos diferentes procesos como una problematización inspirada en Theatrum Philosophicum, Foucault instiga: “¿Cuál es la respuesta a la pregunta? El problema. ¿Cómo resolver el problema? Desplazando la pregunta. El problema escapa a la lógica del tercer excluido, pues él es una multiplicidad dispersa (...)" (Foucault, 2005, p. 246). La problematización es, pues, un proceso de producción que no se resuelve por la idea cartesiana ni por la negatividad hegeliana, pues es una afirmación múltiple. "Es preciso pensar el problema, más que preguntar y responder dialécticamente” (Foucault, 2005, p. 26).

Es posible pensar en cierta forma de interrogación que conduce teórico-metodológicamente: ¿cómo el objeto de estudio, en un momento dado, puede ser problematizado a través de una relación entre el poder institucional y de ciertos estados mixtos del saber metodológico?

Se trata, entonces, de modos de preguntar a cada formación histórica, creando un campo de posibilidades de donde emergen las problemáticas. En esta línea, la escritura del texto busca una estética como configuración de la experiencia, en que colectiviza lo aprehendido, no a través de una sucesión cronológica, sino que lo intenta por otros modos de interrogar y narrar. Los datos no se recopilan y almacenan, sino emergen a partir de lo que la investigación interroga. Este juego de fuerzas conduce a pensar en una problematización de los desplazamientos, en lugar de encontrar en los precedentes un origen como respuesta.

\section{Consideraciones finales}

En el legado de Matteart hay una problematización de la cultura cotidiana en un trabajo de epistemología histórica, metodologicamente muy cerca de lo que hace Foucault con la genealogia que analisa el saber como estratégia del poder. 
El poder se busca en su contexto práctico, analizando el dispositivo histórico de las condiciones políticas de posibilidad de los discursos, no el origen, sino la procedencia. "La parte genealógica del análisis se centra en la serie de formación efectiva del discurso, se trata de aprehenderlo en su poder de afirmación. Y entiendo, por eso, no un poder que se oponga a la negación, sino el poder de constituir dominios de objetos" (Foucault, 2001 p. 71, traducción propria).

Son relacionados los diferentes entendimientos de lo que es método y de lo que es científico. Es la constitución de cómo estos campos de conocimiento se articulan para poder pensar en la diferenciada emergencia histórica de estos dos objetos: método y científico. De la misma forma que el método es construido como universal, una determinada concepción de científico también viene siendo producida históricamente, desde el siglo XVIII. Es decir, si tomados en su perspectiva histórica, tanto método y científico son construcciones de las prácticas sociales en determinados momentos que producen continuamente saberes sobre ellos. Se busca entonces dividir la expresión "método científico", tan naturalmente utilizada, e incluso banalizada en el pensamiento contemporáneo.

Es en este sentido que la desnaturalización consiste "en comprender que las cosas no pasan de las objetivaciones de prácticas determinadas, cuyas determinaciones deben ser expuestas a la luz, ya que la conciencia no las concibe (...)" (Veyne, 1982, p. 162). Se percibe que en la constitución de diferentes prácticas que los programas de postgrado ofrecen en sus micropolíticas hay, también, diferentes agenciamientos que van produciendo diferentes "rostros" y, por lo tanto, diferentes objetos. Al final: "Todo gira alrededor de esa paradoja, que es la tesis central de Foucault y la más original: lo que se hace, el objeto, se explica por lo que fue el hacer en cada momento de la historia; nos equivocamos cuando pensamos que hacerlo, la práctica, se explica a partir de lo que se hace" (Veyne, 1982, p.164).

Lo que se intenta es problematizar los enunciados dirigidos a un saber metodológico que, bajo regímenes de verdad instituidos, acaban 
por normalizar determinados modos de existencia, siguiendo las pistas de que "el genealogista es un poco adivino, filósofo del futuro" (Deleuze, 1976, p. 45).

Así hizo Mattelart que, en vez de pensar los saberes sobre una moral de la razón sobre el pensamiento, buscó por medio de otras construcciones, creaciones e invenciones, pensar diferentes modos de sensibilidad, diferentes modos de vivir, existir, percibir, sentir, en fin, diferentes maneras de estar y existir en el mundo. Sin embargo, estas afirmaciones de la vida en sus potencialidades son todavía vistas como fuera de las tradicionales normalizaciones del saber-poder metodológico, porque no están presentes en los modelos concordantes con la "esencia" de lo que es método científico empírico académico.

\section{Referencias}

Butler, J. (2001). ¿̨ué es la crítica? Un ensayo sobre la virtud de Foucault. En Instituto Europeo para Políticas Culturales Progresivas. Mayo 2001. Recupera- do de http://eipcp.net/transversal/o8o6/butler/es.

Castro Orellana, R. (2004). Ética para un Rostro de Arena. Michel Foucault y el cuidado de la libertad, Madrid: Universidad Complutense de Madrid.

Cusicanqui, S. C. (2010). Ch’ixinakax utxiwa. Una reflexión sobre prácticas y discursos descolonizadores. Buenos Aires: Tinta Limón.

Dreyfus, H., Rabinow, P. (2001). Sobre la genealogía de la ética: una visión de conjunto de un trabajo en proceso. En Michel Foucault: más allá del estructuralismo y la hermenéutica. Buenos Aires: Nueva Visión.

Deleuze, G., Parnet, C. (1998). Diálogos. São Paulo: Escrita.

Deleuze, G. (2000). Lógica do sentido. São Paulo: Perspectiva. . (1976). Nietzsche e a filosofia. Rio de Janeiro: Editora Rio.

Foucault, M. (2010). Ética, sexualidade, política. Ditos \& escritos V. Rio de Janeiro: Forense.

Foucault, M. (1995). ¿Qué es la crítica? [Crítica y Aufklärung]. En Daimon, Revista de Filosofía, $\mathrm{N}^{\circ}$ 11. Universidad de Murcia. (2012), Vigilar y castigar: Nacimiento de la prisión. España: Biblioteca Nueva. . (2010). O governo de si e dos outros. São Paulo: Martins Fontes. 
. (2005).Theatrum Philosophicum. En Foucault, M. Ditos \& Escritos II - Arqueologia das ciências e história dos sistemas de pensamento (pp. 230-254). Rio de Janeiro: Forense Universitária. . (2010). Para uma moral do desconforto. En Foucault, M. Ditos e Escritos Vol. VI: repensar a política (pp. 279-284). Rio de Janeiro: Forense Universitária.

. (2010). Prefácio à Transgressão. En Foucault, M. Ditos e Escritos, v.III. Rio de Janeiro: Forense Universitária.

Dorfman, A., \& Mattelart, A. (1972). Para leer al Pato Donald: Comunicación de masas y colonialismo. Siglo XXI Editores.

Guattari, F.; Rolnik, S. Micropolítica: cartografias do desejo. 2.ed. Petrópolis: Vozes, 1986.

Gros, F. (2010) Caminhar, uma filosofia. São Paulo: É Realizações.

Mattelart, A. \& Vitalis, A. (2015). De Orwell al cibercontrol. Barcelona: Gedisa.

Mattelart, A. (1981). "Hacia una Teoria Crítica de la comunicación", Memorias de la Semana Internacional de la Comunicación, Bogotá, Ed. Unv. Javeriana. . (1991). La publicidade. Barcelona: Paidós Ibérica. . (1994). Comunicação mundo: história das ideias e das estratégias. Petrópolis: Vozes. . (1996). A invenção da comunicação. Lisboa: Instituto Piaget.

Mattelart, A. \& Mattelart, M. (1997). História de las teorías de la comunicación. Barcelon: Ediciones Paidós Ibérica.

Maldonado, A. E. \& Fígaro R. (2020). A Vertente Mattelart como pensamento comunicacional crítico. Matrizes: V.14 - No 3 set./dez. p. 7-25.

Maldonado, A. E. \& Castro, E. L. (2019). Investigación crítica de la comunicación en América Latina: diálogos con la vertiente Mattelart. Ecuador: Ediciones Ciespal.

Maldonado, A. E. (1999). Teorias críticas da comunicação: o pensamento de Armand. Intexto, Porto Alegre: UFRGS, v. 2, n. 6, p. 1-23. . (2008). A perspectiva transmetodológica na conjuntura de mudança civilizadora em inícios do século XXI. In: A. E. Maldonado; J. Bonin; Nísia Rosário (org.). Perspectivas metodológicas em comunicação: desafios na prática investigativa. João Pessoa: Editora UFPB.

. (2006). Práxis teórico-metodológica na pesquisa. In: A. E. Maldonado et al. Metodologias de pesquisa em comunicação/Olhares trilhas e processos. Porto Alegre: Sulina. 
. (2003). Explorações sobre a problemática epistemológicas no campo das ciências da Comunicação. In: LOPES, Maria Immacolata Vassalo. (Org.). Epistemologia da Comunicação. São Paulo: Loyola.

. (2002). Produtos midiáticos, estratégias e recepção/A perspectiva transmetodológica. In: Revista Ciberlegenda, UFF, № 9 .

Proust, M. (1998). Em busca del tiempo perdido. El tiempo recobrado. Madrid, Alianza.

Rimbaud, A. (1995). Poesía completa. Rio de Janeiro: Topbooks.

VEYNE, P. (1982). Como se escreve a história: Foucault revoluciona a história. Brasília, DF: UnB. 